\title{
Timing analysis of the core of the Crab-like SNR G21.5-0.9
}

\author{
N. La Palombara and S. Mereghetti \\ Istituto di Fisica Cosmica "G. Occhialini", via Bassini 15, 20133 Milano, Italy
}

Received 15 October 2001 / Accepted 18 December 2001

\begin{abstract}
The Crab-like SNR G21.5-0.9 was observed in the X-ray band (0.5-10 keV) by the XMM-Newton satellite for over $100 \mathrm{ks}$. The large effective area of the EPIC instrument has allowed us to perform a deep search for pulsations from the central core of G21.5-0.9. No pulsations were found with upper limits on the pulsed fraction between $7.5 \%$ and $40 \%$ (depending on frequency and energy range).
\end{abstract}

Key words. ISM: individual: G21.5-0.9 - supernova remnants - X-rays: ISM

\section{Introduction}

Almost $5 \%$ of the $\sim 225$ known supernova remnants (SNRs) in our galaxy are classified as "Crab-like" or "plerionic" (Green 2000). From the spatial point of view, they are characterized by compact, centre-filled radio and X-ray morphology (Weiler \& Panagia 1978). These SNRs show featureless power-law spectra, with a relatively flat spectral index in the radio regime $\left(\alpha_{\mathrm{r}} \sim 0.0-0.3\right)$ and a steeper one at shorter wavelengths, which are typical of synchrotron processes.

These morphological and spectral characteristics are explained by the presence of a central pulsar, which injects high energy electrons that suffer synchrotron radiation losses as they diffuse through the surrounding magnetic field (see, e.g., Reynolds \& Chanan 1984).

G21.5-0.9 shows many characteristics of the Crab-like remnants. Both in the radio band (Morsi \& Reich 1987) and at X-ray energies (Becker \& Szymkowiak 1981) its emission is centrally peaked. Evidence for a non-thermal $\mathrm{X}$-ray spectrum was also indicated by GINGA observations (Asaoka \& Koyama 1990).

The available measurements of the neutral hydrogen absorption give a distance of $\sim 4.8 \mathrm{kpc}$ (Becker \& Szymkowiak 1981). The radio luminosity of G21.5-0.9 is $\sim 1.8 \times 10^{34} d_{5}^{2} \mathrm{erg} \mathrm{s}^{-1}$ (Morsi \& Reich 1987), i.e. a factor $\sim 9$ smaller than that of the Crab, but its $\mathrm{X}$-ray luminosity is a factor $\sim 100$ less, therefore the $L_{\mathrm{X}} / L_{\mathrm{r}}$ ratio is significantly lower.

$\mathrm{X}$-ray observations performed with the Chandra satellite detected a compact central core of $\sim 2^{\prime \prime}$ in size, at the center of the more extended synchrotron nebula of $\sim 30^{\prime \prime}$

Send offprint requests to: N. La Palombara,

e-mail: nicola@ifctr.mi.cnr.it radius (Slane et al. 2000). The central core, which is spatially resolved, most likely marks the position of the pulsar powering G21.5-0.9. Also a fainter, more extended "halo" (radius $\sim 2^{\prime}$ ) was detected with Chandra. This was tentatively interpreted as the outer "shell" formed by the expanding ejecta and the passage of the supernova-driven blastwave (Slane et al. 2000). However, a recent XMMNewton observation shows that also the halo has a nonthermal spectrum; it is probably a low surface brightness extension of the plerionic nebula (Warwick et al. 2001). However, this interpretation is not supported by radio data, since no significant radio emission has yet been detected at such a large distance from the source core (Bock \& Wright 2001).

Up to now no pulsed emission has been detected in the radio or $\mathrm{X}$-ray energy range from the putative neutron star at the center of G21.5-0.9 (Frail \& Moffet 1993; Kaspi et al. 1996; Biggs \& Lyne 1996; Slane et al. 2000). In this paper we report the results of a sensitive timing analysis on data provided by four XMM-Newton observations.

\section{Observations and data analysis}

During April 2000, G21.5-0.9 was observed four times by the XMM-Newton mission as one of the calibration targets. A $\log$ of the observations is given in Table 1 . The source was on-axis in the first observation and $\sim 10^{\prime}$ offaxis in the following ones; in each case the accumulated exposure time was $\sim 30 \mathrm{ks}$. The results reported by Warwick et al. (2001) were based only on the on-axis observation.

In all the observations the source was imaged by the three focal plane $C C D$ cameras (MOS1, MOS2 and $P N$ ) of the EPIC instrument (Turner et al. 2001; Strüder et al. 2001). Each of the $M O S$ cameras provides an effective area 
Table 1. Summary of the observations.

\begin{tabular}{|c|c|c|c|c|}
\hline $\begin{array}{c}\text { Start time (UT) } \\
\text { April } 2000\end{array}$ & $\begin{array}{c}\text { Off-axis angle } \\
\text { arcmin }\end{array}$ & $\begin{array}{c}\text { Exposure } \\
\mathrm{ks}\end{array}$ & $\begin{array}{c}\text { MOS frame } \\
\mathrm{s}\end{array}$ & $\begin{array}{c}\text { PN frame } \\
\mathrm{ms}\end{array}$ \\
\hline $7,12 \mathrm{~h} 35 \mathrm{~m}$ & 0.2 & 30 & 2.6 & 200 \\
$9,12 \mathrm{~h} 22 \mathrm{~m}$ & 10.3 & 29 & 2.6 & 73 \\
$11,12 \mathrm{~h} 26 \mathrm{~m}$ & 10.4 & 29 & 2.6 & 73 \\
$15,12 \mathrm{~h} 26 \mathrm{~m}$ & 10.2 & 29 & 2.6 & 73 \\
\hline
\end{tabular}

of $\sim 600 \mathrm{~cm}^{2}$ at $1.5 \mathrm{keV}$, and covers the energy range 0.2 $10 \mathrm{keV}$; for the $P N$ camera the corresponding values are, respectively, $\sim 1400 \mathrm{~cm}^{2}$ and $0.15-15 \mathrm{keV}$. All the instruments were operated with the medium filter. The $P N$ camera worked in Extended Full Frame mode in the first observation, with a $C C D$ frame time of $200 \mathrm{~ms}$, and in standard Full Frame mode in the following ones, with a frame time of $73 \mathrm{~ms}$; both MOS cameras used the Full Frame mode in all the observations, with a frame time of $2.6 \mathrm{~s}$.

The first step of our data analysis was the event selection. For each of the four observations and for each of the three cameras we extracted all the events, with energy between 1 and $10 \mathrm{keV}$, from a circular region with radius of $25^{\prime \prime}$ centered at the peak of the $\mathrm{X}$-ray emission $\left(\mathrm{RA}=18 \mathrm{~h} 33 \mathrm{~m} \mathrm{33.8s}, \mathrm{DEC}=-10^{\circ} 34^{\prime} 6^{\prime \prime}(\mathrm{J} 2000)\right)$. This radius contains $\sim 80 \%$ of the photons for a point source. We only considered events with pattern in the range $0-12$ and $0-4$ for, respectively, the $M O S$ and the $P N$ camera, in order to reject the non $\mathrm{X}$-ray events due to cosmic rays and cosmetic defects.

The times of the selected events were converted to the Solar System barycenter with the Reconstructed Orbit Files provided by the XMM Survey Science Center. These events were still tagged with discrete arrival times, corresponding to the readout times of the individual CCD frames: these arrival times were "randomized" by subtracting a random value between 0 and the relevant frame time from the original times.

On these data we performed search for periodicities based on Fourier analysis (see, e.g., van der Klis 1989). A Fourier power spectrum was computed for each of the instruments and observations and examined for the presence of peaks above a threshold corresponding to a chance probability of $10^{-3}$ of being exceeded in the absence of a signal in a single spectrum. No significant peak was found.

To increase the detection sensitivity we repeated the same analysis on the sum of the individual spectra. To this aim, due to the three different CCD frame times (0.073, 0.2 and $2.6 \mathrm{~s}$ ), we had to consider three different frequency ranges for the data analysis (Table 2):

- for $\nu<0.1923 \mathrm{~Hz}=(2 \times 2.6 \mathrm{~s})^{-1}$, we summed the power spectra of all the observations and instruments;

- for $0.1923 \mathrm{~Hz}<\nu<2.5 \mathrm{~Hz}=(2 \times 0.2 \mathrm{~s})^{-1}$, we summed the power spectra of only the $P N$ data of the four observations;
- for $2.5 \mathrm{~Hz}<\nu<6.85 \mathrm{~Hz}=(2 \times 0.073 \mathrm{~s})^{-1}$, we summed the power spectra of the $P N$ data of the three off-axis observations.

Again, no statistically significant peak was found in the summed power spectra. We also repeated the whole procedure for two distinct energy ranges, $1-3.5 \mathrm{keV}$ and 3.5-10 keV, again without any significant pulsed signal.

To compute the upper limits on the source pulsed fraction, in the assumption of a sinusoidal pulse shape, we followed the procedure described in van der Klis (1989), taking into account the relevant correction pointed out by Vaughan et al. (1994). Finally, we had to correct the resulting upper limits to take into account the fraction of unpulsed flux due to the nebular emission. We based this correction on the results of the Chandra observation (Slane et al. 2000), showing that at least $92 \%$ of the flux within $30^{\prime \prime}$ is of diffuse origin. Thus we obtained the upper limits (99.9\% confidence level) on the pulsed fraction of the neutron star emission given in Table 2, where we also report the number of counts used in the analysis.

\section{Discussion}

Our upper limits can be compared with those obtained by some recent works based on X-ray data of G21.5-0.9. Note that the values reported by Warwick et al. (2001), $3.5 \%$ and $5.5 \%$ (respectively for $\mathrm{MOS}+\mathrm{PN}$ and $\mathrm{PN}$ only data), refer to the total flux within an extraction radius of $8^{\prime \prime}$, including the nebular emission. Our results for the onaxis observation alone (the one used by these authors) are similar, but we reached a better sensitivity in the summed power spectra (corresponding to a factor $\sim 4$ greater exposure time).

For instrumental reasons, our search was limited to periods longer than $146 \mathrm{~ms}$. Although it is clearly possible that the pulsar in G21.5-0.9 has a shorter period, we note that Safi-Harb et al. (2001), from energetic considerations, estimated a period of $P=0.144\left(I_{45} /\left(\dot{E}_{37} \tau_{3}\right)\right)^{1 / 2} \mathrm{~s}$ (where $I=10^{45} I_{45} \mathrm{~g} \mathrm{~cm}^{2}$ is the moment of inertia, $\dot{E}=10^{37} \dot{E}_{37} \mathrm{erg} \mathrm{s}^{-1}$ is the spin-down energy loss and $\tau=\tau_{3} \times(3 \mathrm{kyr})$ is the pulsar characteristic age $)$. For reasonable values of $\tau$ and $\dot{E}$, such a period falls in the range we could explore with EPIC. The same authors analyzed five data sets (total $75 \mathrm{ks}$ ) obtained with the Chandra $H R C$ instrument. They report an upper limit of $16 \%$, without quoting the confidence level and, presumably, referring to the total flux within $2^{\prime \prime}$. 
Table 2. Upper limits on the pulsed fraction (99.9\% c.l.).

\begin{tabular}{|c|c|c|c|c|c|c|}
\hline Frequency range & \multicolumn{2}{|c|}{$1-10 \mathrm{keV}$} & \multicolumn{2}{c|}{$1-3.5 \mathrm{keV}$} & \multicolumn{2}{c|}{$3.5-10 \mathrm{keV}$} \\
$\mathrm{Hz}$ & $\%$ & counts & $\%$ & counts & $\%$ & counts \\
\hline$<0.19$ & $6.2-7.5$ & 522142 & $9.1-11.0$ & 300332 & $12.4-13.5$ & 221810 \\
$0.19-2.5$ & $15.1-17.1$ & 275543 & $19.5-22.1$ & 154231 & $24.2-27.3$ & 121312 \\
$2.5-6.8$ & $24.6-30.1$ & 170434 & $32-39.2$ & 98105 & $33.3-40.8$ & 72329 \\
\hline
\end{tabular}

The "canonical" picture of plerionic supernova remnants is based on young, energetic neutron stars with short rotation periods, such as the Crab pulsar $(P=33 \mathrm{~ms})$ or the recently discovered pulsar in $3 \mathrm{C} 58(P=66 \mathrm{~ms}$, Murray et al. 2001). However, other results show that also sensitive searches for slower pulsars are relevant: there are in fact relatively young pulsars with long periods. Besides the well known example of PSR B1509-58 in the SNR G320.4-01.2 ( $P=150 \mathrm{~ms})$, other recent findings include the $325 \mathrm{~ms}$ pulsar in the SNR Kes 75 (Gotthelf et al. 2000), and PSR J1119-6127 ( $P=407 \mathrm{~ms}$, Camilo et al. 2000). which however does not have a bright synchrotron nebula.

Acknowledgements. We wish to thank the Italian Space Agency (ASI) for its financial support to this work.

\section{References}

Asaoka, I., \& Koyama, K. 1990, PASJ, 42, 625

Becker, R. H., \& Szymkowiak, A. E. 1981, ApJ, 248, L23

Biggs, J. D., \& Lyne, A. G. 1996, MNRAS, 282, 691
Bock, D. C. J., Wright, M. C., \& Dickel, J. R. 2001, ApJ, 561, L203

Camilo, F., et al. 2000, ApJ, 541, 367

Frail, D. A., \& Moffett, D. A. 1993, ApJ, 408, 637

Gotthelf, E. V., et al. 2000, ApJ, 542, 37

Green, D. A. 2000, A Catalogue of Galactic Supernova Remnants, Cambridge UK, available at http://www.mrao.cam.ac.uk/surveys/snrs/

Kaspi, V. M., et al. 1996, AJ, 111, 2028

Morsi, H. W., \& Reich, W. 1987, A\&AS, 69, 533

Murray, S. S. et al. 2001, [astro-ph/0108489]

Reynolds, S. P., \& Chanan, G. A. 1984, ApJ, 281, 673

Safi-Harb, S., et al. 2001, ApJ, 561, 308

Slane, P., et al. 2000, ApJ, 533, L29

Strüder, L., et al. 2001, A\&A, 365, L18

Turner, M. J. L., et al. 2001, A\&A, 365, L27

van der Klis, M. 1989, in Timing Neutron Stars, NATO ASI Ser.C, ed. H. Ögelman, \& E. P. van den Heuvel (Dordrecht: Kluwer), 262, 27

Vaughan, B. A., et al. 1994, ApJ, 435, 362

Warwick, R. S., et al. 2001, A\&A, 365, L248

Weiler, K. W., \& Panagia, N. 1978, A\&A, 70, 419 